\title{
Effectiveness of telephone counselling by a pharmacist in reducing mortality in patients receiving polypharmacy: randomised controlled trial
}

\author{
Jennifer Y F Wu, Wilson Y S Leung, Sophie Chang, Benjamin Lee, Benny Zee, Peter C Y Tong, Juliana C N Chan
}

\begin{abstract}
Objective To investigate the effects of compliance and periodic telephone counselling by a pharmacist on mortality in patients receiving polypharmacy.

Design Two year randomised controlled trial.

Setting Hospital medical clinic.

Participants 502 of 1011 patients receiving five or more drugs for chronic disease found to be non-compliant at the screening visit were invited for randomisation to either the telephone counselling group $(n=219)$ or control group $(n=223)$ at enrolment 12-16 weeks later.

Main outcome measures Primary outcome was all cause mortality in randomised patients. Associations between compliance and mortality in the entire cohort of 1011 patients were also examined. Patients were defined as compliant with a drug if they took $80-120 \%$ of the prescribed daily dose. To calculate a compliance score for the whole treatment regimen, the number of drugs that the patient was fully compliant with was divided by the total number of prescribed drugs and expressed as a percentage. Only patients who complied with all recommended drugs were considered compliant (100\% score). Results 60 of the 502 eligible patients defaulted and only 442 patients were randomised. After two years, 31 (52\%) of the defaulters had died, 38 (17\%) of the control group had died, and $25(11 \%)$ of the intervention group had died. After adjustment for confounders, telephone counselling was associated with a $41 \%$ reduction in the risk of death (relative risk $0.59,95 \%$ confidence interval 0.35 to $0.97 ; \mathrm{P}=0.039$ ). The number needed to treat to prevent one death at two years was 16. Other predictors included old age, living alone, rate of admission to hospital, compliance score, number of drugs for chronic disease, and non-treatment with lipid lowering drugs at screening visit. In the cohort of 1011 patients, the adjusted relative risk for death was $1.61(1.05$ to $2.48 ; \mathrm{P}=0.029)$ and 2.87 (1.80 to $2.57 ; \mathrm{P}<0.001$ ) in patients with compliance scores of $34-66 \%$ and $0-33 \%$, respectively, compared with those who had a compliance score of $67 \%$ or more.

Conclusion In patients receiving polypharmacy, poor compliance was associated with increased mortality. Periodic telephone counselling by a pharmacist improved compliance and reduced mortality.

Trial registration International Standard Randomised Controlled Trial Number Register: SRCTN48076318.
\end{abstract}

\section{Introduction}

Many drugs for chronic conditions-notably cardiovascular disease-reduce morbidity and mortality in controlled clinical trial settings. ${ }^{2}$ They may be less effective in clinical practice, however, because of poor compliance-only half of patients who receive polypharmacy are fully compliant. ${ }^{34}$ Although interventions such as telephone or postal reminders from pharmacists improve compliance, their effect on clinical outcome is not known. ${ }^{5-8}$ We investigated whether periodic telephone counselling by a pharmacist to reinforce compliance and ensure continuity of care reduced mortality in patients receiving polypharmacy and whether compliance was associated with mortality.

\section{Methods}

\section{Healthcare setting in Hong Kong}

Hong Kong has a heavily subsidised healthcare system. About $16 \%$ of the general tax revenue is used to finance the Hospital Authority, which governs all 48 public hospitals, 52 hospital based specialised clinics, and 21 community based general outpatient clinics. Together, these provide over $95 \%$ of acute and chronic medical care to the population of 6.8 million. Hence, most patients with chronic diseases are treated in publicly funded clinics where patients pay only a nominal fee averaging HK\$100-200 (£7-14, €10-20, US\$13-26) at each visit. This fee includes the cost of drugs, which are dispensed on site. People in Hong Kong usually visit their family doctors for minor ailments and rely on hospital healthcare teams to monitor chronic conditions.

\section{Participants}

Our study was a two year, prospective, randomised, controlled study conducted at the specialist medical clinics of the Prince of Wales Hospital in Hong Kong with a catchment population of 1.2 million. This is a 1200 bed regional hospital and its medical unit runs 22 outpatient clinics, with more than 2000 patient visits each week. Clinically stable patients are followed up at intervals of two to four months, and each consultation lasts 10-15 minutes. We recruited patients between October 1998 and June 1999. One pharmacist (JYFW), assisted by clinic nurses, screened all records of patients attending the medical clinics the day before their visit.

The inclusion criterion was prescription of five or more drugs on at least two consecutive visits to the clinic. We assessed

The structured questionnaire is on bmj.com 


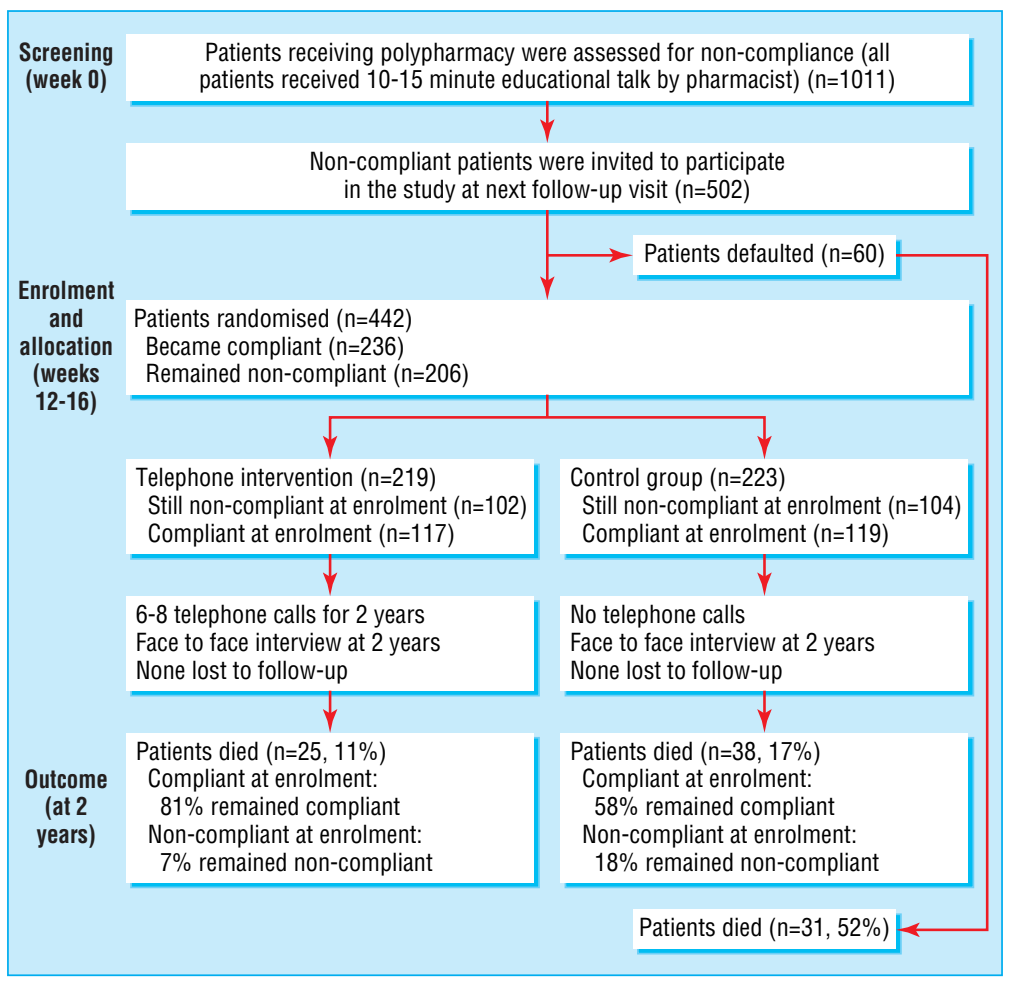

Fig 1 Flow of patients through the trial

compliance only for drugs that were prescribed on a chronic basis. The same pharmacist assessed eligible patients for compliance before their medical consultations. We invited noncompliant patients (see later) for randomisation at the next follow-up visit, usually 12-16 weeks later (fig 1).

We excluded patients who spoke non-Cantonese dialects or a different language or had conditions that prevented effective communication (for example, patients who were deaf, mute, or had dementia or other psychological disorders). We also excluded patients who lived in nursing homes with supervised treatment. All eligible patients gave informed consent.

\section{Screening and definition of compliance}

During the interview, patients were given drug samples to help them remember the regimen prescribed at their last visit and to help the pharmacist validate their information. Using a structured questionnaire (see appendix on bmj.com), the pharmacist asked the patient to describe their regimen by drug dosages (number of pills), frequency (number of doses), and number of pills taken at different times of the day. They were asked whether they had missed any doses; changed their regimens in terms of doses, frequency, and timing; or had drugs left over. This information was checked against the dispensing information on the Health Authority's clinical management system. These questions gave the pharmacist a reasonable impression of the patient's degree of compliance and the consistency of their information. We defined patients as compliant with a drug if they had taken $80-120 \%$ of the prescribed daily dose. To calculate a compliance score for the whole treatment regimen, we divided the number of drugs that the patient was fully compliant with by the total number of prescribed drugs and expressed it as a percentage. A patient who complied with all prescribed drugs had a compliance score of $100 \%$, whereas one who complied with only three of the six drugs had a compliance score of $50 \%$. We assessed compliance at the screening visit, randomisation visit, and two year follow-up visit. Our pharmacist gave patients a 10-15 minute educational talk on correct usage of drugs and they were given education materials at the screening visit.

\section{Enrolment and randomisation}

At the enrolment visit, we reassessed eligible patients for compliance. The pharmacist was blinded to the randomisation codes, which were computer generated by our statistician and sealed in envelopes labelled with consecutive numbers. The envelopes were opened by the clinic nurse in an ascending manner, and patients were allocated to the intervention or control group.

\section{Intervention}

Patients allocated to the intervention group received a 10-15 minute telephone call from our pharmacist at the midpoint between clinic visits throughout the study period. The pharmacist asked about the patient's treatment regimens; clarified any misconceptions; explained the nature of any side effects; reminded patients of their next clinic appointment; and reinforced the importance of compliance with treatment and relevant aspects of self care, such as diet, exercise, and self monitoring. Because of frequent changes of attending doctors, information was not fed back to the clinic staff, although patients were encouraged to report all side effects, self initiated changes in regimen, or concerns to their doctors at their next follow-up visit. Patients in the control group received no telephone interventions.

\section{Outcome measures and data collection}

The primary endpoint was the time from randomisation to death from any cause. Other endpoints included changes in the rate of admission to hospital, number of emergency room visits, and hospital stay in the two years before and after the screening visit, as well as changes in compliance. Baseline data included patients' demographics, socio-cognitive functioning, and use of 
drugs at the screening visit. Drug compliance was assessed at the screening visit, enrolment visit, and at the end of the two year study period. Since 1995, all important clinical data including hospital admissions, emergency room attendance, and dispensing information are computerised and can be accessed by healthcare workers from the clinical management system. Two senior doctors confirmed the causes of death by reviewing medical records and examining death certificates with an accountability of $100 \%$.

\section{Statistical analysis}

We used SPSS version 9 for all analyses. The Student's sample $t$ test and $\chi^{2}$ test, where appropriate, were used to compare groups.

For the primary analysis of the randomised group, we used an intention to treat analysis. We used Kaplan-Meier analysis to compare death rates and expressed the results as relative risks and 95\% confidence intervals. A forward stepwise algorithm was used in a multivariate Cox proportional model to select predictors of mortality, including non-intervention by the pharmacist, age, sex, severity of disease (rate of admission to hospital and number of visits to the emergency room), compliance scores, number of concomitant drugs, and use of life saving drugs at screening visit.

In the secondary analysis of the entire cohort of patients, we used Cox proportional hazard regression and Kaplan-Meier analysis to obtain hazard ratios and $95 \%$ confidence intervals for mortality in patients, according to their baseline compliance scores. Patients whose compliance score was $67 \%$ or more were used as the referent group. A two sided $\mathrm{P}$ value $<0.05$ was considered significant.

\section{Estimation of sample size}

Most of the patients receiving polypharmacy had cardiovascular diseases or multiple risk factors with an estimated annual mortality of $10 \% .^{9}$ In a seven year observational study, the death rate of hypertensive patients with type 2 diabetes who received structured care from a specialist in diabetes and a nurse was $8 \%$ compared with $24 \%$ in those who received usual clinic care. Continuity of care by the same team, reinforcement of compliance, and periodic assessments were the key features of the structured care model. ${ }^{10}$ Based on these local figures, we hypothesised that intervention by a pharmacist would reduce mortality in these high risk patients by $50 \%$, from $20 \%$ to $10 \%$, in two years (equivalent to a hazard ratio of 2.12). To achieve $80 \%$ power with a two sided $\alpha$ level of $0.05,200$ patients were needed in each group to obtain at least 60 events. Allowing for a $10 \%$ rate of attrition, we randomised 440 non-compliant patients. If we assumed a $50 \%$ rate of non-compliance, we needed to recruit 1067 patients to confirm this rate with a precision of $3 \%$; this would also give enough non-compliant patients for randomisation to confirm the effects of intervention on mortality.

\section{Results}

\section{Primary analysis of randomised cohort}

\section{Characteristics of the patients}

We interviewed 1011 patients taking multiple drugs for chronic conditions (49\% male; mean age 71 years, SD 10, range 34-96; mean number of drugs taken 5.9, 1.2, 5-14). Of these, 502 were non-compliant and were invited to participate in our study; however, 60 patients defaulted at the subsequent visit. At enrolment, 236 patients were compliant and equal numbers were randomised to each group (fig 1). Baseline characteristics were
Table 1 Data at screening visit in patients receiving polypharmacy randomised to intervention group with periodic telephone counselling by a pharmacist or control group. Values are number (\%) unless stated otherwise

\begin{tabular}{|c|c|c|}
\hline Variable & $\begin{array}{l}\text { Control group } \\
(\mathrm{n}=223)\end{array}$ & $\begin{array}{l}\text { Intervention group } \\
(\mathrm{n}=219)\end{array}$ \\
\hline \multicolumn{3}{|l|}{ Sociodemographic characteristics } \\
\hline Mean (SD) age (years) & $70.5(11.1)$ & $71.2(9.4)$ \\
\hline Male & $107(48)$ & $108(49)$ \\
\hline Ever smoked & $91(41)$ & $100(46)$ \\
\hline \multicolumn{3}{|l|}{ Patients with different compliance scores: ${ }^{*}$} \\
\hline $0-33 \%$ & $30(14)$ & $33(15)$ \\
\hline $34-66 \%$ & $72(32)$ & $65(30)$ \\
\hline$\geq 67 \%-99 \%$ & $121(54)$ & $121(55)$ \\
\hline $100 \%$ & 0 & 0 \\
\hline Mean (SD) compliance score (\%) & $55.5(26.5)$ & $65.9(21.0)$ \\
\hline Administered drugs themselves & $202(91)$ & $207(95)$ \\
\hline Unable to read labels & $37(17)$ & $37(17)$ \\
\hline Lived alone & $33(15)$ & $30(14)$ \\
\hline \multicolumn{3}{|l|}{ Comorbidities } \\
\hline Mean (SD) No of drugs for chronic illnesses & $5.9(1.2)$ & $6.0(1.3)$ \\
\hline Mean (SD) No of daily doses* & $13.9(6.4)$ & $13.2(6.3)$ \\
\hline $\begin{array}{l}\text { Median (25th and } 75 \text { th centiles) No of emergency } \\
\text { room visits each year (for } 2 \text { years before } \\
\text { screening visit) }\end{array}$ & $1.0(0.0,4.0)$ & $2.0(1.0,3.0)$ \\
\hline $\begin{array}{l}\text { Median (25th and } 75 \text { th centiles) No of admissions } \\
\text { to hospital each year (for } 2 \text { years before } \\
\text { screening visit) }\end{array}$ & $1.0(0.0,2.0)$ & $1.0(0.0,2.0)$ \\
\hline $\begin{array}{l}\text { Median (25th and } 75 \text { th centiles) No of days in } \\
\text { hospital each year (for } 2 \text { years before screening } \\
\text { visit) }\end{array}$ & $4.0(0.0,11.0)$ & $4.0(0.0,11.0)$ \\
\hline
\end{tabular}

*Patients were defined as compliant with a drug if they took $80-120 \%$ of the prescribed daily dose. To calculate a compliance score for the whole treatment regimen, the number of drugs that the patient was fully compliant with was divided by the total number of prescribed drugs and expressed as a percentage. Only patients who complied with all recommended drugs were considered compliant ( $100 \%$ score).

similar except that the control group had a lower compliance score (table 1) and lower use of lipid lowering and antiplatelet drugs (table 2). Most of the patients were elderly, lived with family members, and had been admitted to hospital several times before. About 13\% of the participants had their drugs sorted or administered (or both) by caregivers (table 1).

\section{Effects of telephone counselling}

We observed all patients for at least two years or until death. The mean follow-up period was 23.2 months (SD 4.5). Each patient in the interventional group had six to eight telephone calls from the same pharmacist between clinic visits. At two years, 38 patients (17\%) had died in the control group compared with 25 $(11 \%)$ in the intervention group (fig 2). Most patients died from cardiovascular events (table 2). After we adjusted for confounding variables, the intervention was associated with a $41 \%$ reduction in the relative risk of all cause mortality and the number needed to treat to prevent one death was 16 .

At enrolment, half of the participants had become compliant after a brief talk by a pharmacist at the screening visit. Of the 236 patients who changed to being compliant, 14 of 117 (12\%) died in the intervention group and 18 of $119(15 \%)$ in the control group. Of the 206 who stayed non-compliant at enrolment, 11 of $102(11 \%)$ died in the intervention group and 20 of 104 (19\%) in the control group. Compliance was reassessed at the end of the two year study period in patients who survived. Fewer patients who were non-compliant at enrolment remained non-compliant at the end of the study in the intervention group than in the control group (7\% (7 of 102) v 18\% (19 of 104), $\mathrm{P}<0.001$ ). More patients who turned compliant at enrolment remained 
Table 2 Drugs used for chronic illnesses (defined according to the British National Formulary) in patients receiving polypharmacy randomised either to telephone intervention by a pharmacist or to the control group. Values are number of patients $(\%)$

\begin{tabular}{|c|c|c|}
\hline Class of drug (system affected) & $\begin{array}{l}\text { Control group } \\
(\mathrm{n}=223)\end{array}$ & $\begin{array}{l}\text { Intervention group } \\
\quad(\mathrm{n}=219)\end{array}$ \\
\hline \multicolumn{3}{|l|}{ At enrolment } \\
\hline Central nervous system & $10(5)$ & $15(7)$ \\
\hline Endocrine system: & $87(39)$ & $93(43)$ \\
\hline Antidiabetic drug & $74(33)$ & $83(38)$ \\
\hline Other & $15(6)$ & $13(6)$ \\
\hline Gastrointestinal system & $39(18)$ & $40(18)$ \\
\hline Gynaecological system and urinary tract & $1(0.4)$ & $0(0)$ \\
\hline Musculoskeletal system & $42(19)$ & $39(18)$ \\
\hline Nutritional supplements & $82(37)$ & $72(33)$ \\
\hline Respiratory system & $61(27)$ & $60(27)$ \\
\hline \multicolumn{3}{|l|}{ Cardiovascular system: } \\
\hline $\begin{array}{l}\text { Angiotensin converting enzyme inhibitor or } \\
\text { angiotensin II antagonist }\end{array}$ & $111(50)$ & $117(53)$ \\
\hline$\alpha$ blocker & $19(9)$ & $16(7)$ \\
\hline$\beta$ blocker & $58(26)$ & $68(31)$ \\
\hline Antiplatelet agent & $97(44)$ & $121(55)$ \\
\hline Calcium channel blocker & $106(48)$ & $94(43)$ \\
\hline Diuretic & $118(53)$ & $119(54)$ \\
\hline Lipid lowering drug & $29(13)$ & $43(20)$ \\
\hline Nitrate & $96(43)$ & $111(51)$ \\
\hline Any drug affecting cardiovascular system & $211(95)$ & $214(98)$ \\
\hline \multicolumn{3}{|l|}{ End of study } \\
\hline \multicolumn{3}{|l|}{ Cardiovascular drugs: } \\
\hline $\begin{array}{l}\text { Angiotensin converting enzyme inhibitor or } \\
\text { angiotensin II antagonist }\end{array}$ & $103(46)$ & $112(51)$ \\
\hline Antiplatelet agent & $102(46)$ & $129(59)^{\star \star}$ \\
\hline Lipid lowering drug & $38(17)$ & $56(26)^{\star}$ \\
\hline
\end{tabular}

compliant in the intervention group than in the control group (81\% (95 of 117) v 58\% (69 of 119), $\mathrm{P}=0.038$ ).

Compared with the two year period before the screening visit, the increase in the use of healthcare resources was greater in the control group than in the intervention group, and reached significance for number of days in hospital each year (median (25th and 75th centiles), $3.0(-2.0,17.5)$ v 0.0 (-4.0, 10.0), $\mathrm{P}=0.018$; median number of hospital admissions each year: 1.0 $(-1.0,2.0) v 0.0(-1.0,2.0), \mathrm{P}=0.316)$. The increase in frequency of visits to the emergency room each year did not differ significantly between groups $(0.0(-1.0,2.0)$ v $0.0(-1.0,2.0)$, $\mathrm{P}=0.203$.

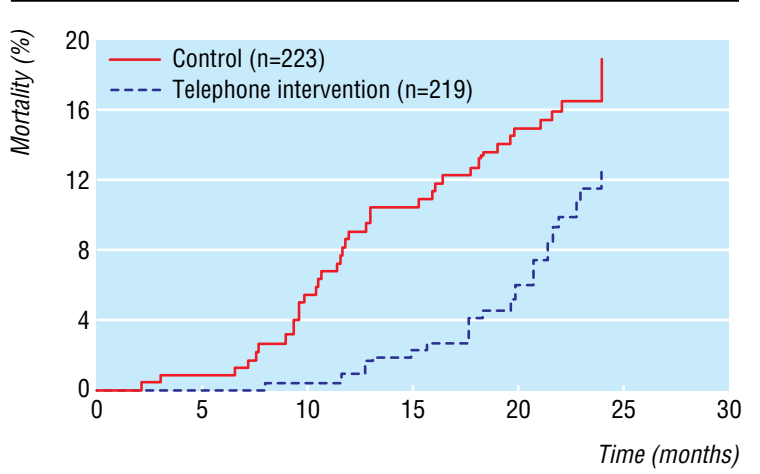

Fig 2 Kaplan-Meier estimates of effect of telephone intervention by a pharmacist on all cause mortality in patients receiving polypharmacy (relative risk for intervention $0.59,95 \%$ confidence interval 0.35 to $0.97, P=0.039$ after adjusting for confounding factors)
Table 3 Primary causes of death in patients receiving polypharmacy randomised either to telephone intervention by pharmacist or to control group. Values are number (\%)

\begin{tabular}{lcc} 
Primary cause of death & $\begin{array}{c}\text { Control group } \\
(\mathbf{n}=\mathbf{2 2 3})\end{array}$ & $\begin{array}{c}\text { Intervention group } \\
(\mathbf{n}=\mathbf{2 1 9})\end{array}$ \\
\hline All causes & $38(18)$ & $25(11)^{\star}$ \\
\hline Cardiovascular events & $20(33)$ & $11(16)$ \\
\hline Renal failure & $3(5)$ & $0(0)$ \\
\hline Cancer & $4(6)$ & $5(8)$ \\
\hline Infection & $9(14)$ & $6(10)$ \\
\hline Other & $2(3)$ & $3(5)$ \\
\hline
\end{tabular}

${ }^{\star} \mathrm{P}<0.05$.

\section{Effect of drug use and other factors on mortality}

More patients in the intervention group than in the control group received antiplatelet and lipid lowering drugs at baseline and at two years (table 2). Sixty three patients died and table 3 shows the primary causes of death. Patients who died were less likely to take $\beta$ blockers (6 (10\%) v $121(32 \%), \mathrm{P}<0.0001)$ and more likely to take diuretics $(47(75 \%)$ v $189(50 \%), \mathrm{P}<0.0001)$ and drugs that affect the musculoskeletal system (20 (32\%) $v 60$ $(16 \%), \mathrm{P}=0.005)$ or respiratory system $(26(41 \%)$ v $94(25 \%)$, $\mathrm{P}=0.01)$ at baseline than those who survived. At two years, survivors were more likely to take angiotensin converting enzyme inhibitors or angiotensin II antagonists (196 (52\%) v 19 (32\%), $\mathrm{P}=0.005)$ and lipid lowering drugs $(90 \quad(24 \%)$ v $4(7 \%)$, $\mathrm{P}=0.002)$. After we adjusted for all confounding variables including use of drugs at baseline, only old age, living alone, lack of pharmacist's intervention, number of drugs for chronic conditions, length of hospital stay during the study period, baseline compliance scores, and not taking lipid lowering drugs at screening visit were independent predictors for death (table 4).

\section{Secondary analysis of the entire cohort}

\section{Effects of compliance on mortality}

Most of the cohort of 1011 patients were taking drugs for cardiovascular disease, followed by drugs for diabetes. We identified 5920 dosage regimens, with once daily being the most frequent $(54 \%)$, followed by twice daily $(32 \%)$, three times daily $(9 \%)$, and four times daily $(5 \%)$. The compliance scores for these regimens were $85.8 \%, 78.4 \%, 74.4 \%$, and $52.8 \%$, respectively. Figure 3 shows the risk of death in patients with compliance scores of $0-33 \%$ and $34-66 \%$ compared with those who had a score of $67 \%$ or more. After we adjusted for number of visits to the emergency room and number of hospital admissions in the past 24

Table 4 Regression analysis of all cause mortality at two years in patients receiving polypharmacy randomised to either intervention (telephone counselling) or control groups after adjusting for sociodemographic characteristics, comorbidities, use of drugs, and compliance at screening visit

\begin{tabular}{lcc} 
Independent variables & Relative risk $\mathbf{( 9 5 \% ~} \mathbf{~ I I )}$ & P value \\
\hline Age & $1.05(1.02$ to 1.08$)$ & 0.001 \\
\hline Living alone & $3.56(1.28$ to 9.88$)$ & 0.015 \\
\hline Telephone counselling by pharmacist & $0.59(0.35$ to 0.97$)$ & 0.039 \\
\hline No of concomitant drugs & $1.27(1.05$ to 1.54$)$ & 0.015 \\
\hline Length of hospital stay (past 24 months) & $1.02(1.01$ to 1.03$)$ & $<0.001$ \\
\hline Baseline drug compliance score & $0.98(0.97$ to 0.99$)$ & $<0.001$ \\
\hline Taking lipid lowering drugs at baseline & $0.28(0.10$ to 0.82$)$ & 0.020 \\
\hline
\end{tabular}

Dependent variable: death $=1$

Independent variables: age, sex, ability to read labels, living arrangement, rate of admission to hospital, emergency room attendance, hospital stay two years before and during the two year follow-up period, smoking status, pharmacist's telephone counselling, number of concomitant drugs, compliance score, and use of lipid lowering drugs, antiplatelet agents, angiotensin converting enzyme inhibitors, and angiotensin II antagonists at screening visit. 


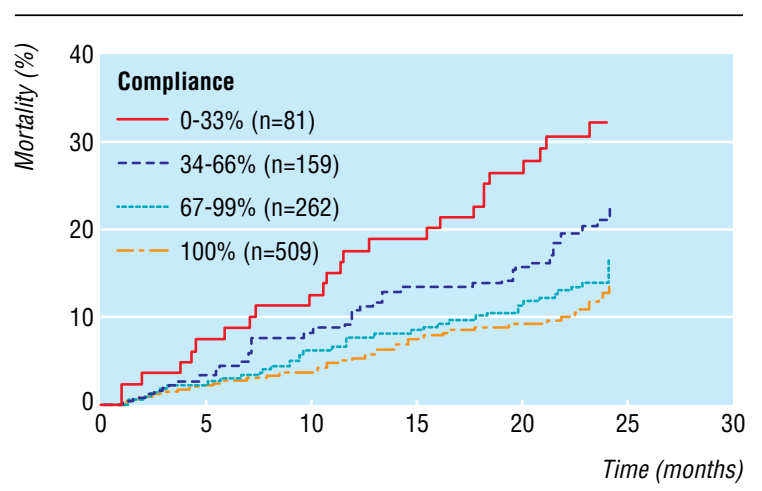

Fig 3 Kaplan-Meier estimates for 1011 patients receiving polypharmacy according to compliance score at the screening visit. Relative risks and $95 \%$ confidence intervals for death in patients with compliance scores of $0-33 \%$ and $34-66 \%$ were 2.9 (1.8 to $4.6, \mathrm{P}<0.001)$ and 1.8 (1.1 to 2.7, $\mathrm{P}=0.0098)$ compared with those who had a score of $67 \%$ or more

months, age, and number of concomitant drugs, hazard ratios were 2.87 (confidence interval 1.80 to $4.57, \mathrm{P}<0.001$ ) and 1.61 (1.05 to $2.48, \mathrm{P}=0.029)$ for patients with compliance scores of $0-33 \%$ and $34-66 \%$. The other independent risk factors were age $(1.05,1.03$ to $1.07, \mathrm{P}<0.001)$ and rate of admission to hospital (1.19, 1.14 to 1.25$), \mathrm{P}<0.001)$.

\section{Clinical outcomes in the defaulters}

Clinical and sociodemographic characteristics of the 60 defaulters were similar to those of the randomised patients (data not shown). At two years, 31 of the 60 defaulters had died (hazard ratio 6.8 (4.4 to 10.4$), \mathrm{P}<0.0001$ compared with randomised patients). Defaulters had a lower compliance score (mean 48\%, $\mathrm{SD} 29 \% v 62 \%, 24 \% ; \mathrm{P}=0.019)$; they also had a higher number of admissions to hospital each year (median (25th and 75th centiles), $2.0(1.0,5.0) v 1.0(0.0,2.0), \mathrm{P}=0.001)$, number of visits to the emergency room each year $(2.0(1.0,6.0) v 2.0(1.0,4.0)$, $\mathrm{P}=0.007)$, and number of days in hospital each year (15.0 (3.3, 50.5) v $4.0(0.0,13.3), \mathrm{P}=0.001)$ at baseline than non-defaulters. At two years, the number of visits to the emergency room increased $(3.4(0.0,10.7)$ times a year), as did rates of admission to hospital $(3.2(0.0,15.0)$ times a year) and length of hospital stay $(46.6(0.0,221.0)$ days a year) (all $\mathrm{p}=0.001$ compared with the two years before the screening visit).

\section{Discussion}

In this single centre, randomised, controlled study, telephone counselling by a pharmacist improved compliance, reduced mortality, and reduced use of healthcare resources in patients receiving polypharmacy. The beneficial effects on mortality remained significant after we controlled for confounding factors, including minor differences in use of drugs at baseline. Apart from improved compliance, the telephone calls may have triggered discussions on the patients' health and led to greater awareness and more proactive attitudes towards health in the patients or their carers, or both. Our pharmacist also encouraged patients to discuss any problems with their healthcare teams; this might have influenced the drug regimens that they were prescribed, resulting in better compliance and tolerance in this group. Because of their comorbidities and frequent changes in hospital healthcare teams, we could not evaluate the effects of control of disease processes and changes in drugs on clinical outcomes based on review of medical records alone. In most public healthcare settings, patients are managed by different healthcare teams. Regular counselling by our pharmacist might have improved the continuity of care. Such a rapport might be sufficient to alleviate patients' concerns, which would otherwise have led to discontinuation of drugs or visits to the emergency room. Evidence now shows that multidisciplinary care that emphasises compliance, periodic assessments, use of appropriate drugs, and attainment of treatment goals could substantially reduce complication rates. ${ }^{10-14}$

\section{Assessment of compliance and its associations with mortality}

Compliance can be measured by many methods, such as self reports, pill counts, biological markers, and electronic monitoring devices, all of which have strengths and limitations. ${ }^{15}{ }^{16}$ Given the complexity of these regimens, we relied on direct interviews together with patients' accounts, computerised information, and pill samples to obtain a reasonable estimate of compliance. These interviews provided invaluable insights into the reasons why patients did not follow treatment regimens, which helped our pharmacist target counselling at specific problems, such as attitude or insufficient skills or knowledge. ${ }^{17}$ On the basis of this assessment method, half of our participants were non-compliant, and non-compliance increased with complexity of the treatment regimens. ${ }^{18}$ In the cohort of 1011 patients, we found that the risk of death increased with the degree of non-compliance, which supports the validity of our compliance score.

\section{Importance of reinforcment to changing behaviour}

After brief counselling by our pharmacist at the screening visit, half of the non-compliant patients eligible for randomisation had become compliant at enrolment. Because periodic reinforcements are needed to prevent relapse and maintain behavioural changes, these patients were randomised despite the improvement in compliance. ${ }^{19} 20$ At two years, patients who remained non-compliant at enrolment and received no further intervention had the highest death rate. Non-compliant patients in the intervention group had similar death rates to patients who turned compliant at enrolment but did not receive further reinforcement. Thus, continuous support is needed to change behaviour and reinforce positive health behaviours, which over time could be translated into major clinical benefits.

Similarly, defaulters had the highest rates of mortality and morbidity. Several studies have reported high rates of failing to keep appointments or claiming repeat prescriptions. ${ }^{21}{ }^{22}$ Although we did not systematically examine the reasons for default, review of case notes and telephone calls showed that some of these patients could not return for follow-up because they were in hospital or an institution. Frequent changes in drugs or lack of social support might have exacerbated their non-compliance. Some defaulters might have felt well and not seen the need for long term follow-up, whereas some might have defaulted owing to forgetfulness, leftover drugs, or competing priorities, incuding difficulty in obtaining leave from work. In Hong Kong, patients who miss three consecutive appointments may be taken off the clinic list and will need a new referral by the family doctor for the case to be reopened. Irrespective of the reasons for default, these defaulters are a target group for intervention.

\section{Limitations of our study}

As with most health service research, blinding was not possible because the intervention was complex and caregivers were involved. ${ }^{23}$ To minimise these potential biases, we used predefined inclusion and exclusion criteria, preassigned computer generated codes opened by an independent person in the randomisation process, and a structured questionairre to 
document compliance. We also used hard endpoints such as all cause mortality and rates of admission to hospital-measured by review of death certificates and hospital records-to reduce detection bias with adjustment for potential confounders. Despite the encouraging results, we need to examine other sociological, cognitive, psychological, and behavioural determinants that may influence compliance and clinical outcomes. These results need to be replicated using a multicentre, randomised strategy, and such a study is being carried out in patients with chronic heart failure. ${ }^{24}$

Thanks to Philip KT Li and MF Lau for their assistance. We thank all staff at the PWH Li Ka Shing Specialist Medical Clinic and Albert Cheung, our statistician, for study design and analyses. We thank Bryan Williams, University of Leicester, for critically appraising our paper. This paper is dedicated to the late Julian AJH Critchley for his inspiration and support.

Contributors: JCNC conceptualised, designed, and coordinated the study and finalised the manuscript. JYFW conducted the study, collected and analysed all data, and drafted the manuscript. WYSL helped analyse data and write the paper. PCYT, BL, SC, and BZ gave advice and edited the manuscript. All authors read and approved the manuscript. JCNC is guarantor.

Funding: Hong Kong Government Health Care and Promotion Fund (HSRC/HCPF grant 226103) and MSD international grant.

Competing interests: JCNC and PCYT are investigators in clinical trials and research programmes sponsored by MSD. JCNC is also a member of the MSD Worldwide Diabetes Advisory Board.

Ethical approval: Chinese University of Hong Kong clinical research ethics committee.

1 Vijan S, Hayward RA. Pharmacologic lipid lowering therapy in type 2 diabetes mellitus: background paper for the American College of Physicians. Ann Int Med 2004;140:650-8.

2 Vijan S, Hayward RA. Treatment of hypertension in type 2 diabetes mellitus: blood pressure goals, choice of agents, and setting priorities in diabetes care. Ann Intern Med 2004;138:593-602.

3 Grol R, Grimshaw J. From best evidence to best practice: effective implementation of change in patient's care. Lancet 2003;362:1225-30.

4 Colley C, Lucas L. Polypharmacy: the cure becomes the disease. J Gen Intern Med 1993:8:278-83.

5 Friedman R, Kazis L, Jette A, Smith M, Stollerman J, Torgerson J, et al. A telecommunications system for monitoring and counseling patients with hypertension. Impact on medication adherence and blood pressure control. Am J Hypertens 1996;9:285-92.

\section{What is already known on this topic}

Patients who receive polypharmacy have low levels of compliance

The complexity of the treatment regimen is associated with non-compliance, and non-compliance is associated with increasing risk of death in a stepwise manner

\section{What this study adds}

Periodic telephone counselling by a pharmacist of non-compliant patients receiving polypharmacy improves compliance with treatment and reduces mortality and use of healthcare resources
6 Robinson J, Conroy C, Wickemeyer W. A novel telephone-based system for management of secondary prevention to a low-density lipoprotein cholesterol $\leq 100$ $\mathrm{mg} / \mathrm{dl}$. Am J Cardiol 2000;85:305-8.

7 Guthrie R. The effects of postal and telephone reminders on compliance with pravastatin therapy in a national registry: results of the first myocardial infarction risk reduction program. Clin Ther 2001;23:970-80.

8 Haynes RB, McKibbon KA, Kanani R. Systematic review of randomised trials of interventions to assist patients to follow prescriptions for medications. Lancet 1996;348:383-6.

9 So WY, Chan NN, Tong PCY, Chow CC, Chan WB, Ng MCY, et al. Effect of RAAS inhibition on survival and renal outcomes in 3737 Chinese type 2 diabetic patients. Hypertension 2004;44:294-9.

10 So WY, Tong PCY, Ko GT, Leung WYS, Chow CC, Yeung VTF, et al. The effects of protocol driven care versus usual outpatient clinic care on survival rates in type 2 diabetic patients. Am J Managed Care 2003;9:606-15.

11 Aubert RE, Herman WH, Waters J, Moore W, Sutton D, Peterson BL, et al. Nurse case management to improve glycemic control in diabetic patients in a health maintenance organisation. A randomised, controlled trial. Arch Intern Med 1998;129:605-12.

12 Gaede P, Vedel P, Larsen N, Jensen GVH, Gunnar J, Parving H-H, et al. Multifactorial intervention and cardiovascular disease in patients with type 2 diabetes. $N$ Engl J Med 2003;348:383-93.

13 Discher C, Klein D, Pierce L, Levine A, Levine T. Heart failure disease management: impact on hospital care, length of stay and reimbursement. Congest Heart Fail 2003;9:77-83.

14 Leung WYS, So WY, Tong PCY, Chan NN, Chan JCN. The effects of structured care by a pharmacist-diabetes specialist team on renal outcomes in type 2 diabetic patients with nephropathy and renal impairment. Am J Med 2005;118:1414.

15 Cramer JA. Overview of methods to measure and enhance patient compliance. In: Cramer JA, Spilker B, eds. Patient compliance in medical practice and clinical trials. New York: Raven Press, 1991:3-10.

16 Rudd P, Byyny RL, Zachary V, LoVerde ME, Titus C, Mitchell WD, et al. The natural history of medication compliance in a drug trial: limitations of pill counts. Clin Pharmacol Ther 1989;46:169-72.

17 Prochaska JO, Velicer WF, Rossi JS, Goldstein MG, Marcus BH, Rakowski W, et al. Stages of change and decisional balance for 12 problem behaviors. Health Psychol 1994;13:39-46.

18 World Health Organization. Defining adherence. In: Adherence to long-term therapies: evidence for action. Geneva: WHO, 2003:3-5. www.who.int/chronic_conditions/ adherencereport/en/ (last accessed 18 Jul 2006).

19 Muhlhauser I, Berger M. Patient education-evaluation of a complex intervention. Diabetologia 2002;45:1723-33.

20 Schwarzer R. Social-cognitive factors in changing health-related behaviors. Current Directions in Psychological Science 2001;10:47-51.

21 Smith C, Yawn B. Factors associated with appointment keeping in a family practice residency clinic. J Fam Pract 1994;38:25-9.

22 Hamilton W, Hopkins U. Survey on unclaimed prescriptions in a community pharmacy. JAMA 1997;37:341-5.

23 Day S, Altman D. Statistics notes: blinding in clinical trials and other studies. BMJ 2000;321:504.

24 Grancelli H, Varini S, Ferrante D, Schwartzman R, Zambrano C, Soifer S, et al, for GESICA Investigators. Randomised trial of telephone intervention in chronic heart failure (DIAL): study design and preliminary observations. J Card Failure 2003;9:172-9.

(Accepted 29 June 2006)

doi 10.1136/bmj.38905.447118.2F

Department of Medicine and Therapeutics, Chinese University of Hong Kong, Prince of Wales Hospital, Shatin, Hong Kong SAR

Jennifer Y F Wu pharmacist

Wilson Y S Leung pharmacist

Peter C Y Tong associate professor

Juliana C N Chan professor of medicine and therapeutics

Department of Pharmacy, Prince of Wales Hospital

Benjamin Lee pharmacist

School of Pharmacy, Chinese University of Hong Kong

Sophie Chang assistant professor

Centre for Clinical Trials, Chinese University of Hong Kong

Benny Zee director

Correspondence to:J C N Chan jchan@cuhk.edu.hk 